\title{
Smarte Schüler/innen in der Smart City? Zur Bedeutung und Adaption eines Zukunftskonzepts in Schulbüchern
}

\author{
*gruenberg@em.uni-frankfurt.de, Goethe-Universität Frankfurt \\ ** dorsch@geo.uni-frankfurt.de, Goethe-Universität Frankfurt
}

eingereicht am: 02.02.2016, akzeptiert am: 17.03.2016

\begin{abstract}
In dieser Studie werden das Smart City-Konzept vorgestellt und Fähigkeiten von Smart Citizens, wie z. B. Mündigkeit und Partizipation, deduziert. Wir zeigen auf, dass diese Fähigkeiten aktueller denn je sind und warum Schüler/innen auf diese Herausforderungen vorbereitet werden müssen. Dazu werden curriculare Dokumente sowie Schulbücher quantitativ und qualitativ analysiert. Als Ergebnis zeigt sich, dass Schulbücher das Smart City-Konzept nicht ganzheitlich aufgreifen, aber indirekte Anknüpfungspunkte ermöglichen, die zuletzt diskutiert werden.
\end{abstract}

Keywords: Smart City, Mündigkeit, Partizipation, Reflexion, Schulbuchanalyse

\section{Smart pupils in the smart city? On the meaning and adaption of a future concept in textbooks}

In this study, we will present the smart city concept and deduce abilities, e. g. maturity and participation, of smart citizens. We argue that these abilities are more relevant than ever and why pupils need to be prepared for this challenge. To this end we apply a quantitative and qualitative analysis to curricular documents and textbooks. As a result it becomes clear that the textbooks do not look at the smart city concept in its entirety, but offer indirect links which we discuss at the end.

Keywords: smart city, maturity, participation, reflection, textbook analysis

\section{$1 \quad$ Einleitung}

Ein Großteil der heutigen Schüler/innen wird in Städten leben, die sich in den nächsten Jahren in ihrer Organisation grundsätzlich von heutigen urbanen Räumen unterscheiden werden. Die Verbreitung schneller und kostengünstiger Kommunikations- und Informationstechnologie, die umfassende Verfügbarkeit mobiler Endgeräte und die Möglichkeit großflächig Daten zu erheben, förderten in den letzten Jahren die Entwicklung und Verbreitung des Smart City-Konzepts. Dieser neue Ansatz in der Stadtplanung zeichnet sich dadurch aus, dass die innerstädtischen Verwaltungsabläufe durch die Nutzung von Big Data und die Partizipation ihrer Einwohner/innen in höchstem Maße effizient und - im Idealfall - orientiert an den Nutzerinnen und Nutzern organisiert werden (vgl. Caragliu et al. 2011). Angesichts gegenwärtiger Herausforderungen, beispielsweise der globalen Erwärmung und demographischer Entwicklungen, erscheint die Idee von einer Stadt, die durch effiziente und bedarfsori- entierte Energieversorgung klimaneutral ist und dank eines flexiblen und durch Nutzer/innen gesteuerten Verkehrskonzepts, das z. B. älteren Menschen ermöglicht mobil zu sein, äußerst verlockend. Tatsächlich hat das Konzept, das auf unterschiedliche Bereiche der städtischen Verwaltung abzielt, bereits in über 100 Städten weltweit Anwendung gefunden. Viele weitere Städte befinden sich im Transformationsprozess oder haben Teile des Konzepts übernommen (ABI Research 2011).

Die heutige Generation von Schülerinnen und Schülern wird in diesen Städten in der Zukunft leben, sie aufbauen und durch ihre aktive Partizipation gestalten oder zumindest zeitweise dorthin pendeln, um z. B. einzukaufen oder zu arbeiten. Diesbezüglich eröffnen sich auch neue Herausforderungen für die geographische Bildung und es rücken zwei Fragen in den Blickpunkt: Zum einen, welche Fähigkeiten mündige Bürger/innen in der Smart City besitzen müssen, und zum anderen, inwiefern der Unterricht in der Schule sie auf diese neuen Herausforderungen vorbereitet. 
In Zweifel steht nicht, dass die künftigen smarten Bürger/innen die Technik beherrschen, die für Partizipationsprozesse genutzt wird, wie beispielsweise Web 2.0-Anwendungen und digitale Kartendienste. Die Jugendlichen sind mit den technischen Aspekten der neuen Medialität durchaus vertraut. Vielmehr ist entscheidend, ob die Jugendlichen reflektiert mit den Beteiligungsinstrumenten der Smart City umgehen können, ob sie ihre Interessen in den Beteiligungsprozessen eigenständig äußern können, sich der Konstruktion medialer räumlicher Darstellungen bewusst sind und - nicht zuletzt - auch die Auswirkungen des Smart City-Konzepts auf gesellschaftliche Prozesse bewerten können. Sie müssen sensibel sein im Umgang mit persönlichen Daten, die in der Smart City als wertvolle Ressource für die vernetzte Verwaltung dienen, und in der Lage sein, die Chancen und Risiken des Smart City-Konzepts kritisch zu reflektieren. Letztlich spiegeln die genannten Anforderungen wichtige Aspekte des Bildungsziels der Mündigkeit wieder, das in der Bildungstheorie seit Jahrhunderten einen prominenten Platz innehatte, in den letzten Jahren der output-orientierten Bildungsforschung jedoch aus den Augen verloren ging und auch in den curricularen Dokumenten der Lehramtsausbildung durchgängig vernachlässigt wurde (Dorsch et al. 2016).

Hier setzt unser Beitrag an: Zunächst identifizieren wir vor dem Hintergrund der mediendidaktischen Konzepte des Spatial Citizenship-Ansatzes und der strukturalen Medienbildung Fähigkeiten, die in der Smart City erforderlich sind, um in ihr mündig partizipieren zu können. Die Frage nach der Umsetzung in den Unterrichtsstunden ist empirisch nur schwer $\mathrm{zu}$ beantworten. Stattdessen untersuchen wir Schulbücher, die ein wesentliches Instrument des Unterrichts darstellen, um Hinweise zu bekommen, welche Möglichkeiten der Umsetzung sich für Lehrkräfte eröffnen. Mittels einer quantitativen und qualitativen Inhaltsanalyse hessischer Erdkundebücher für das Gymnasium überprüfen wir, inwiefern die deduzierten Fähigkeiten fokussiert werden und ob das Konzept der Smart City - implizit oder explizit - in ihnen überhaupt aufgegriffen wird. Abschließend stellen wir Ansätze, mit denen das Konzept und seine kritische Reflektion im Unterricht verankert werden könnten, zur Diskussion.

\section{Theoretischer Hintergrund}

\subsection{Smart City-Konzepte}

Die Idee der Smart City stellt ein politisch-strategisches Innovationsprogramm dar, das verspricht die politische und wirtschaftliche Effektivität in Städten mit Hilfe vernetzter Infrastruktur zu verbessern und die soziale, kulturelle und urbane Entwicklung zu fördern (Caragliu et al. 2011; Kaczorowski 2014). Ursprünglich als Zukunftsvision von Technologieunternehmen, wie IBM, Cisco und Siemens, dazu gedacht, für eine möglichst weitläufige Vernetzung und Aufrüstung der Städte in Hard- und Software sowie IT-Sicherheit zu werben, hat sich die Smart City-Idee $\mathrm{zu}$ einem weltweit in vielen Städten angewandten Konzept entwickelt (ABI Research 2011; Kaczorowski 2014). Sie soll Lösungsvorschläge auf städtische Herausforderungen des 21. Jahrhunderts anbieten, wie z. B. Auswirkungen des Klimawandels, den Ausbau erneuerbarer Energien, den demographischen Wandel sowie die zunehmende soziale Ungleichheit innerhalb der Stadtbevölkerung. Die technische Umsetzung des Konzepts erfolgt durch Systemintegration und Vernetzung von Daten (z. B. Verkehrsströme, Energienutzung, Demographie) mittels einer City Data Cloud, den Aufbau eines Sensorsystems, beispielsweise in den Straßen, um Echtzeitdaten zu erhalten, intelligente Verteilungsnetze für die Energieversorgung (smart grid) sowie eine flächendecke Breitbandkommunikation (Schieferdecker 2011).

Caragliu et al. (2011) benennen sechs Bereiche in der Stadt, die durch das Smart City-Konzept tangiert und zugleich davon profitieren würden: Neben den Domänen Umwelt, Mobilität, Wirtschaft und Governance sind dies auch die Bürger/innen selbst und ihre Alltagswelt (z. B. Freizeit, Wohnen, Kultur). Hieraus leiten die Autorinnen und Autoren folgende Definition ab:

\section{"We believe a city to be smart when investments in human and social capital and traditional (trans- port) and modern (ICT) communication infra- structure fuel sustainable economic growth and a high quality of life, with a wise management of na- tural resources, through participatory governance" (Caragliu et al. 2011, 70).}

Neben der oben bereits erwähnten digitalen Vernetzung betont diese Definition - anders als frühere Konzepte - die Investitionen in eine nachhaltige und soziale Stadtentwicklung sowie die Einbindung der Bürger/innen in stadtpolitische Entscheidungen. Diese Partizipation kann über digitale (Karten-) Plattformen stattfinden, auf denen Informationen eingetragen werden: Dies können Mängel in der städtischen Infrastruktur sein, wie beispielsweise defekte Straßenlaterne oder Schlaglöcher in Fahrradwegen. Solche „Mängelmelder" existieren bereits in vielen Städten. Ebenso gibt es Plattformen, die dazu auffordern, Vorschläge für die Stadtentwicklung zu machen, über die online abgestimmt wird und die bei Erfolg in den $\mathrm{Ma}-$ 
gistrat eingebracht werden ${ }^{1}$. Das Smart City-Konzept sieht den Ausbau dieser Plattformen vor. Dies kann nach Ansicht einiger Autorinnen und Autoren nur mit einer verstärkten Transparenz in der städtischen Verwaltung und offenen Daten einhergehen, damit die Bürger/innen über das nötige Wissen verfügen, das sie für die Entscheidungen benötigen (Kaczorowski 2014; Schieferdecker 2011).

Mandl \& Schaner $(2012,193)$ betonen, dass die Entwicklung einer Stadt zur Smart City keine technologische Herausforderung darstelle, sondern vielmehr eine gesellschaftliche: Die Technik sei bereits vorhanden, wie die oben vorgestellten Beteiligungsplattformen zeigen, werde aber oftmals nicht von der Bevölkerung akzeptiert. Dies liege zum einen am fehlenden Willen zur Verhaltensänderung bei den Bürgerinnen und Bürgern, welche die Möglichkeiten der Partizipation nicht ausreichend nutzen, aber auch bei den politischen Akteurinnen und Akteuren, die bereit sein sollten, Entscheidungen an die Bevölkerung abzugeben. Zum anderen würden die technischen Lösungen häufig an den Interessen von Nutzerinnen und Nutzern und gesellschaftlichen Ansprüchen vorbei entwickelt. Kritik wird zudem dahingehend geäußert, dass in vielen bisher umgesetzten Konzepten, Datenschutz kaum eine Rolle spielt (Greenfield 2013; Novy 2015): Daten, ob in Echtzeit von Sensoren aufgezeichnet oder in städtischen Ämtern archiviert, sind die wichtigste Ressource für das Funktionieren und die Effizienzsteigerung einer Smart City (Stichwort: Big Data). Greenfield (2013) kritisiert, dass die technischen Systeme in drei Smart Cities, die er untersucht hat (Songdo City in Südkorea, Masdar-City in Abu Dhabi und PlanIT Valley in Portugal), einzig dazu dienten, die Gewohnheiten der Einwohner/innen zu überwachen und sie zu gängeln. Sennett (2012) stimmt darin überein und warnt davor, dass die durchstrukturierte und sterile Organisation der Smart Cities „urbanes Leben" gefährde, das durch Unsicherheiten geprägt sein müsse. Ihre Bewohner/innen würden dadurch geistig abstumpfen.

\subsection{Mündigkeit in der bildungstheoretischen Diskussion}

Die smarte Stadt bedarf folglich mündiger Bürger/ innen: Einerseits, da sie in zentralen Verwaltungsabläufen die Partizipation und kritische Überprüfung durch die Einwohner/innen erfordert, andererseits, da ein sensibler Umgang mit eigenen Daten nötig ist, die den Behörden als Ressource, beispielsweise für ein effizientes Energie- oder Mobilitätsmanagement, die-

\footnotetext{
z.B. die „Ideenplattform“ der Stadt Frankfurt am Main: https://www.ffm.de/frankfurt/de/ideaPtf/45035
}

nen. Damit die Smart City in ihrer Konzeption funktionieren kann, wird somit das klassische Bildungsziel der Mündigkeit wieder aktuell.

Seine Bedeutung über einen rein rechtlichen Status hinaus erlangt der Mündigkeitsbegriff erst unter den Aufklärern des 18. Jahrhunderts. Immanuel Kant wandelt den Zustand zum Prozess, indem er Mündigkeit mit innerer Reife gleichsetzt, der sich der Mensch im Lauf seines Lebens annähern solle. Es sei seine Entscheidung, ob er sich seines Verstandes bediene oder ob er im Dämmerzustand der Unmündigkeit verbleibe (Sommer 2007). Der Begriff Mündigkeit bewegt sich in seiner Deutungsgeschichte zwischen drei Ebenen: Autonomie, Selbst und Reflexivität. Bereits im traditionellen Verständnis wird mit Mündigkeit die Fähigkeit verknüpft, als Individuum autonom, d. h. eigenständig handeln zu können (Blankertz 1982; Heydorn 1980; Kant 1803; Wolff 1740). Inwieweit wahre Autonomie systembedingt überhaupt erreichbar ist, wird in der jüngeren Literatur diskutiert (Adorno 1979; Gruschka 1994). Das bewusste Wahrnehmen der eigenen Identität - die Selbst-Bestimmung - als zentrale Aufgabe von Bildung und damit elementarer Bestandteil von Mündigkeit, umschreibt die zweite Ebene (Klafki 2007; Kößler 1989; Roth 1971). Reflexivität schließlich hilft dem sich selbst wahrnehmenden Menschen seinen eigenen Standpunkt stetig zu relativieren und an die Unbestimmtheit der Gegenwart anzupassen (Adorno 1969; Jörissen \& Marotzki 2009).

\subsection{Mündigkeit als Leitziel in der Smart City}

Die Verwaltung (Governance) stellt den Bereich dar, der in der Smart City die gravierendsten Unterschiede zu konventionellen Städten aufweist und in denen Bürger/innen den größten Gestaltungsspielraum haben. Sie sind nicht mehr bloß Betroffene, sondern müssen Beteiligte in einer Kultur der Partizipation werden (Kaczorowski 2014; Mandl \& Schaner 2012, 194). Damit sie diese Rolle einnehmen können, müssen die Bürger/innen Fähigkeiten erwerben, die in der Schule bereits angelegt werden sollten. Hierbei lässt sich am Spatial Citizenship-Ansatz anknüpfen (Gryl \& Jekel 2012), der einen Hinweis gibt, welche Fähigkeiten auch für Bewohner/innen der Smart City wichtig sind: Der Spatial Citizen ist in der Lage mit Hilfe digitaler Geomedien gesellschaftliche Diskurse zu initiieren. Seine Fähigkeiten berühren dabei nach Schulze et al. (2015) sechs Kompetenzdimensionen. Neben der Kompetenz digitale Geomedien mündig nutzen zu können, ist der Spatial Citizen reflektiert in Bezug auf räumliche Informationen und ihre Darstellung und fähig, eigene Interessen mit Hilfe über das Internet verbreiteter Geomedien zu kommunizieren und auszuhandeln. Darüber hinaus kann er sich Räu- 
me mündig aneignen, ist sich also der sozialen Konstruktion von Räumen bewusst. Zuletzt ist er motiviert und fähig, das Gemeinwesen aktiv mitzugestalten.

Bezogen auf die herausgestellten drei Aspekte von Mündigkeit - Autonomie, Selbst und Reflexivität - bedeutet dies zunächst, dass die Schüler/innen in ihrem Handeln autonom werden sollen. Dabei sind technische Fähigkeiten nicht zu unterschätzen: So kann die internetbasierten Beteiligungsplattformen nur nutzen, wer die Handhabung digitaler Medien beherrscht. Dabei geht es jedoch nicht nur um den technischen Gebrauch von Web 2.0-Anwendungen im Sinne von Know-what und Know-how, sondern auch um den Konsum, die Produktion, Prosumption und Analyse von (Geo-) Daten (Schulze et al. 2015). Die Gefahr der „digitalen Spaltung“ (Kaczorowski 2014, 88) ist hoch, wenn nur diejenigen das Gemeinwesen bestimmen, die mit den Möglichkeiten des Internets umzugehen wissen und weniger internetaffine und eher einkommens- und bildungsschwache Bevölkerungsgruppen nicht integriert werden. Jörissen \& Marotzki 2009 warnen vor einer "voice inequality", sobald die Wissenskluft in der Gesellschaft zu Partizipationshürden führt.

Das Gemeinwohl darf auch in Zukunft nicht verloren gehen. Die Bürger/innen der Smart City entscheiden darüber, wie sich die Stadt, in der sie wohnen, entwickeln soll und - im Sinne Blankertz (1982) - wie viele sie von den tradierten Paradigmen ihrer Vorfahren übernehmen oder verwerfen wollen. Diese Entscheidungen werden nicht wie bisher Einzelnen auferlegt, sondern - sofern das Konzept der Smart City konsequent umgesetzt wird - von allen getroffen (Crowdsourcing). Sie müssen also im Sinne des Spatial Citizenship-Ansatzes mit Hilfe digitaler (Geo-) Medien eigenständig ihre Interessen kommunizieren und mit anderen Bürgerinnen und Bürgern der Smart City aushandeln können.

Diese eigenen Interessen müssen die Schüler/innen zunächst überhaupt erkennen. Nach Roth (1971) obliege es dem mündigen Individuum, sich seines eigenen Selbst bewusst zu werden. Dilthey (1982) sieht die so genannte „Lebenserfahrung” als Hauptaufgabe des Menschen, durch die er erkunde, welche für ihn die maßgebenden Werte darstellten. Die von Jörissen \& Marotzki (2009) geforderte Einnahme unterschiedlicher Perspektiven im Diskurs, indem beispielsweise die Positionen anderer Beteiligter zu urbanen Problemen nachvollzogen werden, unterstützt ihn dabei, den eigenen Standpunkt in Relation zu anderen zu erkennen. Der Mensch vollbringe aus dem Kontext seines Lebenszusammenhangs ständig eine „wertende Ordnungsleistung“, indem er Menschen, Dinge und Informationen hierarchisch sortiere und somit eine individuelle Welthierarchie herstelle. Durch diese
Biographisierung zeige sich in Form einer „Selbst- und Weltreferenz" eine „gewisse Haltung und Auffassung [...] sich selbst wie auch der Welt gegenüber" (Jörissen \& Marotzki 2009, 36). In der Smart City werden Bürger/innen mit einer hohen Zahl an Entscheidungen und zugehörigen Informationen konfrontiert, die es nach subjektiver Relevanz zu hierarchisieren und in einen biographischen Zusammenhang zu stellen gilt. Nach Auffassung der Vertreter der reflexiven Moderne (vgl. z. B. Giddens 1996) muss sich der Grad der eigenen Reflexivität erhöhen, je weniger der Mensch an Traditionen und soziale Einbettungen gebunden ist. Seine Biographie wird dann anstelle von sozialen Kontexten und Gemeinschaften zum Referenzrahmen (Jörissen \& Marotzki 2009). Die Smart City ist Fortführung dessen, was das Internet bisher an Individualisierung und Flexibilisierung hervorgebracht hat: Der Einfluss der gewählten Stadtverordneten, die in Koalitionen organisiert sind, als traditionelle Instanz der Entscheidung in den Städten, schwindet zu Gunsten individueller Entscheidungsträger/innen in der Crowd.

Indem die Bürger/innen ihre eigenen Sichtweisen auf Umwelt und Raum mittels digitaler Medien, wie z. B. Karten, Blogs, Meinungsforen u. ä., produzieren und kommunizieren, stellen sie den bisher meist durch Expertinnen und Experten vorgenommenen (Raum-) -Deutungen eine eigene Perspektive entgegen, um einen Entscheidungsprozess zu beeinflussen. Bezogen auf die Beteiligungsinstrumente der Smart City, mit deren Hilfe Bürger/innen in Raumplanungsprozessen partizipieren oder einfache Petitionen starten können, gilt es also zunächst die soziale Konstruktion dieser Darstellungen zu beachten: Vergleichbar mit der oben benannten reflexiven Kompetenz, müssen sich die Schüler/innen bewusst sein, dass jegliches Medium, sei es nun eine (digitale) Karte oder ein Foto, lediglich eine begrenzte, sozial konstruierte Perspektive auf die Welt wiedergibt (Harley 1989). Genauso stellt der Gebrauch dieser Medien, mit dem Ziel eigene Interessen zu verdeutlichen, einen Akt der Konstruktion dar, durch die die Nutzer/innen ihreWeltperspektive wiedergeben.

Gleichsam sollte der Umgang mit persönlichen Daten Ziel der kritischen Reflexion sein: Die informationelle Selbstbestimmung ist zwar ein Gut, das nicht erst seit der Entwicklung des Smart City-Konzepts gefordert wird (vgl. Marotzki 2000), in Zukunft jedoch essentiell für ein mündiges Handeln in Städten sein wird. Da der Einsatz von Sensoren, beispielsweise auf Straßen, inzwischen für Städte erschwinglich geworden ist, sind die Kommunen in der Lage großflächig und in Echtzeit Daten aufzunehmen, diese mittels Analytik auszuwerten und Prognosen für zukünftige Entwicklungen aufzustellen. Sobald dieses Big DataKonzept jedoch beginnt persönliche Daten zu tangie- 
ren, sollten Schüler/innen in der Lage sein, dies kritisch zu begleiten: So wurden in Chicago beispielsweise die Informationen zu Empfängerinnen und Empfängern von „Essen auf Rädern“ mit Einkommenssteuerdaten kombiniert, um die Wohnorte hilfsbedürftiger älterer Menschen zu ermitteln, die im Katastrophenfall zu evakuieren sind (Kaczorowski 2014) - ein Vorgang, der trotz guter Absichten zumindest diskussionswürdig ist. Ebenso muss die von Greenfield (2013) und Sennett (2012) angeführte Kontrolle der Bewohner/ innen durch technische Systeme von den Schüler/innen kritisch reflektiert werden.

Es zeigt sich, dass Schüler/innen für ein mündiges Agieren in der Smart City bestimmte Fähigkeiten benötigen, die in der Schule angelegt werden sollten: Bezogen auf den Teilaspekt der Mündigkeit Autonomie müssen Schüler/innen in der Smart City digitale Medien eigenständig nutzen und die eigenen Interessen selbstständig kommunizieren können. Zuvor müssen sie dieser Interessen gewahr werden, indem sie unterschiedliche Perspektiven einnehmen und ihren eigenen Standpunkt - das Selbst - in Relation zu anderen aber auch zur eigenen Biographie erkennen und dadurch eine hierarchische Welt- und Werteordnung herstellen. Der reflexive Umgang mit den Medien der Smart City bedeutet, sich bewusst zu machen, dass die Nutzer/innen durch jegliche Darstellung eine persönliche Perspektive konstruieren. Zuletzt müssen die Schüler/innen in der Lage sein, die technischen Möglichkeiten der Smart City kritisch zu hinterfragen. Hierzu zählen vor allem das vorherrschende Leitbild des „Gläsernen Menschen“ sowie mögliche Überwachungs- und Kontrollmechanismen.

\section{Methodisches Vorgehen}

\subsection{Stichprobe}

Bei der Auswahl des Datenmaterials spielten zwei Aspekte eine ausschlagebende Rolle. Dies war zum einen das föderalistische Bildungssystems in Deutschland, das dazu führt, dass Verlage bundeslandspezifische Schulbücher herausgeben, welche sich an den jeweiligen Curricula orientieren, die sich von Bundesland zu Bundesland bezüglich ihrer inhaltlichen Ansätze unterscheiden (Uhlenwinkel 2013). Hiermit ist der zweite Aspekt, das Curriculum, benannt. Vor diesem Hintergrund haben wir für unsere exemplarische Untersuchung nur relevante Dokumente des Bundeslandes Hessen berücksichtigt. Dies hat u. a. auch den pragmatischen Hintergrund, dass wir an hessischen Schulen tätig sind und somit auch die schulpraktische Anknüpfung gewährleistet wird. Zudem wurden nur Dokumente für den gymnasialen Zweig berücksich- tigt, da in dieser Schulform Geographie bzw. Erdkunde in Hessen noch in jeder Klassenstufe ein eigenständiges Fach ist und die gymnasialen Lehrpläne, im Gegensatz zu den Lehrplänen für Haupt-, Real-, und Gesamtschulen aus dem Jahr 2002, im Jahr 2010 aktualisiert wurden.

Da curriculare Dokumente die Grundlage für die in der Schule eingesetzten Lehrmaterialien sind, haben wir im Rahmen unserer Analyse auch den hessischen Lehrplan für das Fach Erdkunde an Gymnasien sowie das Kerncurriculum für die gymnasiale Sekundarstufe 1 berücksichtigt. Das hessische Kerncurriculum für die Oberstufe konnten wir nicht mit aufnehmen, da dies erst im Frühjahr 2016 veröffentlicht wurde.

Da das Smart City-Konzept ein relativ neuer Ansatz ist, war das zentrale Kriterium für die Auswahl der Schulbücher deren Aktualität. Darüber hinaus wurden nur die Werke von Verlagen berücksichtigt, die alle Klassenstufen (Sekundarstufe I und II) abdecken. Dieses Vorgehen ermöglicht es, ein kongruentes Bild auch im Hinblick auf die Analyse der curricularen Dokumente zu gewinnen. Tabelle 1 zeigt den aktuellen Stand des Schulbuchkatalogs für den gymnasialen Zweig, der alle vom Hessischen Kultusministerium zugelassenen Bücher auflistet (Stand: September 2015).

Die Schulbücher der Verlage Klett und Westermann, d.h. sechs Schulbücher, wurden analysiert. Aus den oben aufgezeigten Gründen wurden die Schulbücher der Verlage Schroedel und Cornelsen nicht berücksichtigt.

\subsection{Analysestrategie}

Ziel der Untersuchung war es herauszufinden, ob und in welchem Umfang das Konzept der Smart City in den curricularen Dokumenten und Schulbüchern thematisiert wird und inwiefern die in Punkt 1 extrahierten Fähigkeiten, welche Schüler/innen in der Smart City benötigen, gefördert werden. Vor diesem Hintergrund basierte die inhaltliche Analyse insbesondere auf der Frage nach der Umsetzung aktueller und reflexionsorientierter Themen bzw. Inhalte, welche die Partizipation der Schüler/innen in den Mittelpunkt stellen und eine Verknüpfung mit dem Smart City-Konzept ermöglichen. Zudem standen Schlagworte wie Digitalisierung und Vernetzung im Fokus.

Der gewählte methodische Ansatz der Inhaltsanalyse berücksichtigte sowohl quantitative als auch qualitative Aspekte und orientierte sich an den Vorgaben des UNESCO Guidebook on Textbook Research and Textbook Revision (Pingel 2010). Die deduktiven Kategorien, die basierend auf dem Smart City-Konzept aufgestellt wurden, bildeten dabei die Grundlage der Stichwortsuche in den oben genannten Dokumenten. Hierzu wurden in einem ersten Schritt in den digitalen 
Tab.1: Zugelassene hessische Schulbücher für das Gymnasium im Fach Erdkunde.

\begin{tabular}{|c|c|c|c|c|}
\hline \multirow{2}{*}{$\begin{array}{l}\text { Schulform } \\
\text { Verlag }\end{array}$} & \multicolumn{2}{|l|}{ Sek 1} & \multicolumn{2}{|l|}{ Sek 2} \\
\hline & Titel & Erscheinungsjahr & Titel & Erscheinungsjahr \\
\hline \multirow[t]{2}{*}{ Klett } & Terra 1, KI. 5+6 & 2012 & Fundamente & 2014 \\
\hline & Terra 2, Kl. 8 & 2012 & & \\
\hline \multirow[t]{2}{*}{ Westermann } & Diercke 1, KI. 5+6 & 2012 & Diercke (Oberstufe) & 2011 \\
\hline & Diercke 2, KI. 8 & 2012 & & \\
\hline \multirow[t]{2}{*}{ Schroedel } & Seydlitz 1, KI. 5+6 & 2013 & Seydlitz & 2009 \\
\hline & Seydlitz 2, KI. 7-9 & 2013 & & \\
\hline \multirow[t]{2}{*}{ Cornelsen } & nicht vorhanden & & Mensch und Raum & 2007 \\
\hline & nicht vorhanden & & & \\
\hline
\end{tabular}

Dokumentenfassungen, welche Text, Aufgaben und Abbildungen beinhalten, folgende Wörter gesucht:

- smart,

- digital,

- intelligent,

- beteilig (umfasst Ergebnisse zu den Wörtern Beteiligung und beteiligen),

- partizip (umfasst Ergebnisse zu den Wörtern Partizipation und partizipieren),

- Stadtplaner/Stadtplanung (umfasst auch Ergebnisse zum Wort stadtplanerisch)

- mündig (umfasst Ergebnisse zu den Wörtern mündige/r und Mündigkeit).

Als Ergebnis konnten relevante Textstellen identifziert und quantifiziert werden, die in einem zweiten Schritt qualitativ untersucht wurden. Wenn eine Kontextualisierung bezüglich der Forschungsfrage nicht möglich war, wie z. B. bei dem Satz „Die Temperaturen können mit handelsüblichen Außenthermometern, Digital-Thermometern bzw. Maximal-MinimalThermometern (Physiksammlung) ermittelt werden" (Latz et al. 2012, 302), wurden die Textstellen nicht weiter berücksichtigt. Relevante Ergebnisse wurden in Textstellen mit "direktem Smart City-Bezug" und Textstellen mit „indirektem Anknüpfungspotential“ differenziert: Textstellen mit direktem Bezug weisen konkrete in der Theorie genannte Aspekte des Smart City-Konzepts auf, Textstellen mit indirektem Bezug greifen Bereiche des Smart City-Konzepts auf, setzen sich inhaltlich aber nicht direkt damit auseinander.

In einem dritten Schritt wurden die Inhaltsverzeichnisse der Schulbücher auf mögliche thematische Zusammenhänge bzw. Bezüge zum Smart City-Konzept untersucht. Dieses Vorgehen diente einerseits der Kontrolle der Schritte 1 und 2, um sicherzugehen, dass die Stichwortsuche alle relevanten Seiten aufzeigte, anderseits orientierte es sich an der in der Schulpraxis von Lehrern/innen durchgeführten Inhaltsprobe mit Hilfe des Inhaltsverzeichnisses, getreu dem Gedanken: „Ich suche etwas zur Smart City. Auf welcher Seite könnte etwas dazu stehen?“. Zu Kontrollzwecken wurden auch die curricularen Dokumente solch einer berufs- und alltagspraktischen Analyse unterzogen. Das gesamte methodische Vorgehen folgt der von Pingel (2010) aufgestellten Regel, dass quantitative und qualitative methodische Ansätze sich gegenseitig ergänzen, um substanzhaltige Ergebnisse zu erhalten.

\section{Ergebnisse und Diskussion}

\subsection{Quantitative Gesamtübersicht}

Die quantitativen Ergebnisse der Stichwortsuche und der anschließenden kontextgebundenen vertiefenden Lektüre liefern erste überblicksartige Resultate und verdeutlichen, dass die Thematik Smart City in den curricularen Dokumenten gar nicht und in den Schulbüchern nur an wenigen Stellen aufgegriffen wird (vgl. Anhang 1). Die zentralen Befunde sind, dass hinsichtlich eines direkten Bezuges der Begriff intelligent siebenmal, der Begriff smart zweimal und der Begriff digital nur einmal gefunden wurde. Bei Textstellen mit einem indirekten Bezug, d.h. Anknüpfungspunkten, ergab die Analyse, dass der Begriff beteilig fünfzehnmal, der Begriff Stadtplanung/ Stadtplaner neunmal und der Begriff partizip dreimal ermittelt werden konnte. Die Textstellen mit direktem und indirektem Bezug werden nachfolgend nach curricularen Dokumenten und Schulbüchern gegliedert, beispielhaft vertiefend analysiert und diskutiert.

\subsection{Curriculare Dokumente}

Die Ergebnisliste der quantitativen curricularen Dokumentenanalyse weist lediglich zwei Anknüpfungspunkte im Lehrplan und zwei weitere im Kerncur- 
riculum auf, die sich auf jeweils unterschiedliche Begriffe beziehen. Die Textstellen, in denen sich Anknüpfungspunkte ergeben, sind allgemein formuliert und beziehen sich auf keinen konkreten Fachinhalt, spiegeln aber die Fähigkeiten wider, die Schüler/innen benötigen, um mündig in einer Smart City agieren zu können. So heißt es beispielsweise im Lehrplan:

\section{S. 7, Didaktisch-methodische Grundlagen}

„Das Fach Erdkunde versetzt die Schülerinnen und Schüler in die Lage, [...] sich verantwortungsbewusst an der Bewältigung von Gegenwartsproblemen und Zukunftsaufgaben zu beteiligen" (Hessisches Kultusministerium 2010).

Das Kerncurriculum konkretisiert die Aufgaben des Faches Erdkunde weitergehend:

\section{S. 11, Kompetenzorientierung und Beitrag des Faches zur Bildung \\ "Unsere Umwelt wird durch Naturkräfte und ge- sellschaftliche Gestaltung geprägt. Deshalb brau- chen wir eine verantwortungsvoll handelnde und global denkende Gesellschaft, die aus mündigen, informierten und handlungsfähigen Bürgerinnen und Bürgern besteht. Es liegt an diesen Bürgerin- nen und Bürgern, die Umwelt verantwortungsvoll und reflektiert mitzugestalten" (Hessisches Kul- tusministerium 2011).}

Der mündige Mensch, wie er in den Werken von Kant, Heydorn, Blankertz oder auch Adorno beschrieben wird, der seine Gesellschaft aktiv mitgestaltet, wird in diesen beiden Dokumenten als Ideal und Ziel der geographischen Schulbildung gesetzt. Die theoretischen Ansätze dieser Autoren bilden somit die Grundlage der curricularen Dokumente.

Die kontextbasierte Lektüre der curricularen Dokumente ergab eine weitaus umfangreichere Liste an Verknüpfungen zur Thematik Smart City. Im Kerncurriculum fanden sich im Bereich der geographischen Urteils- und Kommunikationskompetenz zwei Textstellen, bei denen die Beurteilung der Nachhaltigkeit von Maßnahmen zur Gestaltung des Lebensund Wirtschaftsraumes, das Denken in Alternativen sowie die Beurteilung und Bewertung perspektivischer Aussagen über räumliche Nutzung im Mittelpunkt stehen (Hessisches Kultusministerium 2011). Im Lehrplan kann das Thema Smart City insbesondere in der Einführungsphase, d.h. der ersten Jahrgangsstufe in der Oberstufe, zugeordnet werden: In Form einer Projektarbeit soll eine Raumanalyse zum Thema Stadt bzw. Probleme urbaner Ökosysteme durchgeführt werden. Die dabei unter anderem als Beispiel genannten Schwerpunkte Energie, Wasser, Abwasser,
Abfall, Stadtklima, Smog, Naherholung, Verkehr und Stadtentwicklung lassen sich direkt in Bezug setzen zu den von Caragliu et al. (2011) benannten Domänen der Smart City.

Die curricularen Dokumente eröffnen in der Mittelstufe weitere Anknüpfungspunkte: So stellen die Lernbereiche Strukturwandel und Entwicklungschancen des Rhein-Main-Gebietes, der Megalopolis im Osten der USA sowie der Metropolen in Südamerika Möglichkeiten zur Verfügung, diese Themen mit dem Smart City-Konzept zu verknüpfen.

Diese insgesamt ernüchternden Ergebnisse werfen die Fragen auf, wie die Schulbücher hinsichtlich des Lernbereiches Smart City mit diesen Vorgaben aus Lehrplan und Kerncurriculum umgehen und welche Umsetzungsmöglichkeiten hier angeboten werden.

\subsection{Schulbücher}

Der Begriff smart city wird in keinem Schulbuch verwendet und auch der für Schüler/innen möglicherweise leichter zu verstehende deutsche Ausdruck ,intelligente Stadt" führt zu keinem Ergebnis. Das Wort smart wird in nur zwei Sätzen in den (Oberstufen-) Büchern verwendet. Dies erfolgt jeweils im Rahmen eines Textbausteines zur Beschreibung des Themenfeldes Energieversorgung:

\section{„Die Modernisierung im Sinne der Umsetzung von "intelligenten Stromnetzen“, (Smart Grids) hat das Ziel, Stromerzeugung und Stromverbrauch besser aufeinander abzustimmen" (Brodengeier \& Kor- by 2014,174$)$.}

Es ist davon auszugehen, dass Schüler/innen diese Informationen nicht weiter beachten werden, sofern die Lehrkraft den Begriff „smart grid" nicht ausführlich erläutert und mit zusätzlichem Lehrmaterial ergänzt. Ob dies passiert bleibt fraglich, da das Thema der intelligenten Energieversorgung in keiner Aufgabe aufgegriffen wird.

Die Ergebnisse zu dem Suchbegriff beteilig stammen alle aus den Büchern der Sekundarstufe II und mit einer Ausnahme aus dem jeweiligen Kapitel zur Raumordnung. Im Oberstufenbuch des WestermannVerlags werden Ebenen und Akteurinnen und Akteure - wie Städte, Gemeinden sowie Politiker/innen, Stadtplaner/innen und Bürger/innen - sowie ihre Gestaltungspflichten und -möglichkeiten unter die Lupe genommen. In diesem Zusammenhang fällt in beiden Oberstufenbüchern immer wieder das Wort „Bürgerbeteiligung", jedoch ohne dabei Möglichkeiten der internetbasierten Beteiligungsformen zu erwähnen.

Die Wörter Stadtplaner/Stadtplanung beziehen sich auf die bereits oben angesprochenen Gestal- 
tungsmöglichkeiten, oft verknüpft mit dem Aspekt der Nachhaltigkeit. So wird in Fundamente im Kapitel „Leitlinien der Stadtentwicklung in Europa“ beispielsweise die Smart City „Masdar City“ in der Wüste der Vereinigten Arabischen Emirate thematisiert. Sie dient hier als positives Beispiel für eine ressourcenautarke und klimaneutrale Stadt, so dass ausschließlich die Bereiche Mobilität („elektrobetriebene Kabinenfahrzeuge ersetzen den Autoverkehr") und Energie (,ausschließlich regenerative Energiequellen") aufgegriffen werden (Brodengeier \& Korby 2014, 293). Im Diskurs zum Konzept der Smart City wird „Masdar City“ durchaus kritisch gesehen, wie in Punkt 2 aufgezeigt wurde (vgl. Greenfield 2013; Sennett 2012). Dies wird aber im Schulbuch nicht thematisiert, was daran liegen könnte, dass das Schulbuch "Masdar City" in Zusammenhang mit dem Lernbereich "klimatische Erscheinungen“ thematisiert und diesen fachlichen Kontext nicht verlassen will bzw. die Verknüpfung mit einer kritischen Analyse des Smart City-Konzepts nicht in das Blickfeld der Autorinnen und Autoren gerückt ist.

Das Wort intelligent wird im deutschsprachigen Bereich im Kontext von Smart Cities oft verwendet. Dies spiegelt sich auch in den Schulbüchern wider. Mit Ausnahme einer Textstelle zeigen alle sieben Belege einen direkten thematischen Bezug zum Smart CityKonzept auf. Neben der oben bereits erwähnten Textstelle der intelligenten Energieversorgung verknüpfen die übrigen sechs Textstellen das Wort intelligent mit den Wörtern Haus/Büro, Infrastruktursysteme, Mobilität, Auto, Technik und Kleidung. Sie kommen in den Büchern beider Sekundarstufen vor. Erneut werden zahlreiche Domänen der Smart City tangiert, ohne jedoch das Konzept im Ganzen zu beleuchten oder gar zu reflektieren. Die Autorinnen und Autoren der Schulbücher konzentrieren sich auf durchaus faszinierende Technikdetails, ohne die damit einhergehenden Konsequenzen in den Fokus zu rücken.

Die einzige Textstelle, in der die Wortkombination intelligente Mobilität vorkommt, befindet sich auf der gleichen Seite wie der einzige Beleg des Wortes digital (Brodengeier \& Korby 2014, 484 f.) Erst im Rahmen der tiefergehenden qualitativen Untersuchung dieser Doppelseite zeigte sich das Anknüpfungspotential, was insbesondere durch die Methode der Szenariotechnik eröffnet wird. Zur Methode der Szenariotechnik wird von der thematischen Seite "Zur Zukunft der Städte?" verwiesen, die jedoch durch keinerlei weitere Ergebnisse in der Stichwortsuche identifiziert werden konnte, aber die greifbarsten Anknüpfungspunkte zur Thematik der Smart City bietet. In dem Beitrag über die Zukunft der Städte werden anhand zweier Fallbeispiele zukünftige Herausforderungen urbaner Räume thematisiert (Brodengeier \& Korby
2014, 294 f.). Im Mittelpunkt stehen die Vulnerabilität gegenüber Naturrisiken (Erdbeben, Klimawandel), die am Beispiel Istanbuls dargestellt wird, Folgen der Land-Stadt-Wanderung (Überlastung der urbanen Infrastruktur, Marginalisierung, Segregation) sowie die Problematik der schrumpfenden Städte im ländlichen Bereich. Diese Probleme werden in der Literatur immer wieder auch als Ausgangslage für die Städte genannt, für die das Konzept der Smart City eine mögliche Lösung darstellt (vgl. z. B. Caragliu et al. 2011; Kaczorowski 2014). Das im Schulbuch angebrachte Beispiel Istanbul zeigt als so benanntes „worst-case“-Szenario rein negative Entwicklungen in einer Stadt, die sich scheinbar nicht ausreichend auf ein nächstes großes Erdbeben vorbereitet („das schlimmste Szenario“, „Gefahr...verdrängt", „kein geologisches Gedächtnis"). Die klimaneutrale, nachhaltige Stadtentwicklung wird in den darauf folgenden Erläuterungen als erstrebenswertes Ziel genannt und an einem „,best-case“-Szenario einer fiktiven deutschen Stadt im Jahr 2050 illustriert, das im Auftrag des Bundesforschungsministeriums entworfen wurde. Der Name der Stadt „Morgenstadt“ wird interessanterweise von Kaczorowski $(2014,16)$ als Synonym für die Smart City gebraucht. Auffällig ist die einseitig positive Darstellung dieses Zukunftsszenarios in den Bereichen Energieversorgung („regenerative Quellen“, „von fossilen Brennstoffen unabhängig“, „sicherer"), Mobilität („Elektrofahrzeuge“, „deutlich attraktiver und einfacher zu nutzen") sowie Lebensqualität und wirtschaftlicher Wohlstand („Lebensqualität erhöht“, „Wohlstand der Stadt erhöht“, „Arbeitsplätze in Zukunftstechnologie geschaffen"). Neben der bedenkenswerten Darstellung einer türkischen Stadt als worst-case- und einer deutschen Stadt als best-caseSzenario, ist die damit einhergehende Gegenüberstellung zweier komplett unterschiedlicher Themenfelder - Naturrisiken versus Klimaneutralität - nicht nachvollziehbar bzw. für Schüler/innen verwirrend. Die Aufgabenprogression auf der Doppelseite deckt alle drei Anforderungsbereiche (AB) ab, wenn auch kein inhaltlicher Zusammenhang zwischen den einzelnen Aufgaben besteht, was sich mit der erwähnten Themenzweiteilung begründen lässt. In den ersten beiden Aufgaben sollen die Schüler/innen Maßnahmen zur Verhinderung des worst-case-Szenarios nennen (AB 1) bzw. mit Hilfe ihrer Erkenntnisse aus Istanbul und einer Internetseite Risikofaktoren an einem zweiten Raumbeispiel (Dhaka) darstellen (AB 2). Die dritte und vierte Aufgabe bezieht sich nun auf die nachhaltige Stadtentwicklung: Zunächst sollen die im Szenario dargestellten Lösungen mit den im vorigen Kapitel dargestellten Problemen der Stadtentwicklung verglichen werden. Spätestens hier wird den Schüler/innen auffallen, dass sich das Szenario nur auf die Problem- 
bereiche Mobilität, Klima und Energie bezieht, es also für die Herausforderungen, die im Kapitel darüber hinaus erwähnt werden (z. B. soziale Segregation) oder für die gerade erst geforderte Katastrophenvorsorge, keine Lösung anbietet. Die letzte Aufgabe aus dem dritten Anforderungsbereich fordert die Schüler/innen auf, mit Hilfe des Internets selbst ein Szenario zu entwickeln „zu einem ausgewählten Bereich der Entwicklung Ihrer Schulstadt". Als mögliches Themenbeispiel wird genannt: „Demographischer Wandel - Folgen für die Infrastruktur?". Zum Erlernen der Szenariotechnik wird auf die Methodendoppelseite verwiesen. Diese Aufgabe bietet in ihrer Komplexität ausreichend Freiraum, Aspekte der Smart City aufzugreifen, sofern die Lehrkraft die Schüler/innen durch Material und Hinweise in diese Richtung lenkt.

Die Ergebnisse zeigen, dass die Thematik Smart City in keinem Schulbuch explizit behandelt wird. Nichtsdestotrotz werden die urbanen Problembereiche, für welche die Smart City Lösungen bieten möchte, in allen Schulbüchern immer wieder aufgegriffen. Einzelne der vorgestellten Maßnahmen (z.B. smart grid und intelligente Mobilitätssysteme) werden auch im Rahmen der Diskussion um die Smart City thematisiert. Sie werden in den Schulbüchern jedoch nur am Rande erwähnt und nicht in den Aufgaben aufgegriffen. Eine reflektierte Auseinandersetzung mit dem Thema oder eine Debatte darüber, welche Fähigkeiten die Bürger/innen in der „Morgenstadt“ benötigen, finden nicht statt. Dies spiegelt sich auch in der ergebnislosen Suche nach dem Stichwort mündig bzw. Mündigkeit wider. Einzig auf der Szenarioseite im Buch Fundamente findet sich eine Aufgabe, in der die Schüler/innen ein dargestelltes Zukunftsszenario („Stuttgart 2030“) beurteilen sollen (Brodengeier \& Korby 2014, 485). Angesichts dessen, dass auch hier nur Mobilität als ein „smarter“ Bereich dargestellt wird - dies auch nur im positiven Sinne - und der Position der Seite im fakultativen Methodenteil am Ende des Buches, wird deutlich, welch geringer Stellenwert der Mündigkeit zugewiesen wird.

Die Frage, wieso nun die Thematik Smart City nicht aufgegriffen wird, lässt sich nach Hintermann (2010) folgendermaßen beantworten:

„Welche Inhalte in Schulbücher aufgenommen und wie diese dargestellt werden, ist das Resultat von Ein- und Ausschlussprozessen, die durch Lehrpläne und Approbationsverfahren gerahmt sind und durch persönliche Interessen, Werthaltungen und Schwerpunkte sowie den aktuellen Wissensstand und die fachdidaktische Kompetenz der Schulbuchautorl innen beeinflusst werden" (Hintermann 2010, 4).

Lehrplan und Kerncurriculum bieten, wie gezeigt wurde, durchaus Verankerungsmöglichkeiten, wenn- gleich das Thema explizit nicht genannt wird. Der Begriff Smart City wurde in den 2000er Jahren Gegenstand der wissenschaftlichen Diskussion. Curriculare Dokumente und darauf aufbauende Lehrwerke sind in der Regel durch einen längeren Entstehungsprozess geprägt und spiegeln bei ihrem Erscheinen aktuelle gesellschaftliche Diskurse nur ansatzweise wieder. Ursache für die Nichtberücksichtigung könnte somit zum einen der mangelnde Wissenstand der Autorinnen und Autoren sein, die der Thematik in ihrer fachwissenschaftlichen Ausbildung vermutlich nicht oder nur am Rande begegnet sind. Zum anderen ist das Thema, das in seinen Aspekten der Partizipation, des Datenschutzes und der mündigen Begleitung zahlreiche Inhalte der politischen Bildung aufgreift, aus Sicht der Schulbuchakteurinnen und -akteure kein originär geographisches. Möglicherweise sahen einige Autorinnen und Autoren das Thema daher für den Geographieunterricht als wenig relevant an. Dies umso mehr, wenn man ausschließlich den geistigen Ursprung des Begriffs bei den Technologieunternehmen betrachtet und seine kritische Betrachtung außer Acht lässt. Eine rein positive Darstellung einer Smart City wäre in der Tat eine Form der Werbung für die Technologiekonzerne als Entwickler der smarten Technologie. Genau dies geschieht jedoch in den untersuchten Schulbüchern, wenn auch nur in den erwähnten Domänen Mobilität und Energieversorgung. Die Relevanz des Themas wird indes auch für den Geographieunterricht weiter zunehmen: Der Prozess der Digitalisierung, in dem sich manche Städte bereits befinden, andere ihre Potentiale gerade erst entdecken und weiterentwickeln, ist auf lokaler Ebene angesiedelt, prägt sich aber global aus, da das Smart City-Konzept mittlerweile weltweit angewendet wird.

\section{Ausblick und Fazit}

Die Relevanz, der in dieser Studie behandelten Thematik, wird in den nächsten Jahren stark zunehmen: In einer Studie des „Verbands der Elektrotechnik Elektronik Informationstechnik e.V." (VDE) erwarten $67 \%$ der befragten 1300 Mitgliedsunternehmen, dass bis zum Jahr 2030 die ersten deutschen Smart Cities in einem ,volkswirtschaftlich bedeutenden Umfang" entstanden sein werden (VDE 2014). Die oben aufgezeigten Ergebnisse sind angesichts der Aktualität der Thematik und der aufgezeigten "Trägheit" der untersuchten curricularen Dokumente und Schulbücher zunächst wenig überraschend: Wie so oft liegt es an den Lehrkräften, ob eine aktuelle Thematik im Unterricht behandelt wird, indem sie Lehrpläne und Curricula in geschickter Weise ergänzen und interpretieren. Anknüpfungspunkte für das Konzept der Smart City 
sind in ihnen vorhanden, die von den Autorinnen und Autoren der Schulbücher immerhin ansatzweise genutzt werden: Die zukünftigen Herausforderungen urbaner Räume sind in den analysierten Oberstufenbüchern durchaus ersichtlich. Auch werden Szenarien von Städten vorgestellt, die Lösungsvorschläge zu einigen dieser Problemlagen machen, wie z. B. die im Buch „Fundamente“ geschilderte „Morgenstadt“. Sie können als Grundlage für eine kritisch-reflektierte Diskussion des Konzepts dienen, sofern die Lehrkraft die Szenarien mit Informationen, beispielsweise zu bereits umgesetzten Smart Cities wie „Masdar City“, ergänzt. Diese müssen dann aber ganzheitlich $-\mathrm{d}$. h. auch in den in der Literatur kritisierten Aspekten - präsentiert werden. Nur so kann eine Kontrastierung zwischen der utopischen und erstrebenswerten „Morgenstadt" und der dystopischen Überwachungsstadt „Masdar City“ gelingen. Durch entsprechend fordernde Aufgaben aus dem dritten Anforderungsbereich kann die Reflexion des Smart City-Konzepts angeregt werden. Ebenso ist eine Thematisierung der Smart City bereits in der Mittelstufe sinnvoll und gut umzusetzen, wie folgende Skizzierung einer Lerneinheit zeigt, in der sich die Schüler/innen das Konzept der Smart City erarbeiten und multiperspektivisch reflektieren:

Die Schüler/innen setzen sich im Einstieg in Einzel-, Partner- oder Gruppenarbeit mit den nachfolgenden Aufgabenstellungen auseinander:

- Beschreibt, wie ihr euch unsere Stadt in 15 Jahren vorstellt.

- Nennt die dafür nötigen Entwicklungen.

- Erläutert Möglichkeiten, die ihr a) als die Bürgerl innen der Stadt, b) als Politikerlinnen und c) als Stadtplaner/innen habt, um die Lebensqualität in der Stadt zu verbessen.

Diese Aufgaben können - je nach Erwartungshorizont - sowohl von Schüler/innen der Sekundarstufe 1 als auch 2 bearbeitet werden. Für jüngere Schüler/ innen kann der zu untersuchende Raum auf die Schule begrenzt werden. Abhängig vom Kenntnisstand der Klasse lassen sich die Ergebnisse an dieser Stelle den sechs Bereichen des Smart City-Konzepts zuordnen, um dieses dann einzuführen und zu erläutern. Dabei sollen die Lernenden auch bereits vorhandene digitale Beteiligungsplattformen ausprobieren. Nachdem Schüler/innen ihre eigenen Vorstellungen skizziert haben, bereiten sie in Gruppen Interviews bzw. Fragebögen (ggfs. sogar für unterschiedliche Zielgruppen) vor, um Einstellungen von Bürger/innen zum Smart CityKonzept zu erhalten. Neben der Nennung gegenwärtiger städtischer Probleme und ihrer Lösungen, könnte an dieser Stelle auch die Akzeptanz für technische Innovationen abgefragt werden. Die Ergebnisse soll- ten anschließend ausgewertet und dargestellt werden. Abschließend überlegen bzw. recherchieren die Schüler/innen mögliche Lösungsansätze. In der letzten und vorletzten Phase kann auch ein Interview mit Expertinnen und Experten zusätzliche Erkenntnisse liefern. Die Ergebnisse dieser Lerneinheit stellen die Schüler/ innen in einem Portfolio zusammen. Als begleitende Aufgabe sollen sie ihre eigene Einstellung zum Smart City-Konzept ergründen und die Perspektiven der Interviewpartner/innen reflektieren.

Die Lerneinheit ist geeignet den Blick der Schüler/ innen auf das Leben in der Smart City zu richten, Aufgaben und nötige Fähigkeiten der Akteurinnen und Akteur in ihr herauszuarbeiten und das dahinterstehende Konzept kritisch zu reflektieren. So bereiten sie sich Schritt für Schritt auf die digitale Zukunft der Städte vor und können in der Smart City als mündige Bürger/innen agieren.

\section{Literatur}

ABI Research (2011): \$39.5 Billion will be spent on smart city technologies in 2016. http://www.abiresearch.com/ press/3768 (20.01.2016).

Adorno, T.W. (1969): Stichworte. Kritische Modelle 2. Frankfurt a. M.: Suhrkamp.

Adorno, T.W. (1979): Soziologische Schriften 1. Herausgegeben von RolfTiedemann unter Mitwirkung von Gretel Adorno, Susan Buck-Morss und Klaus Schultz. Frankfurt a. M.: Suhrkamp.

Blankertz, H. (1982): Die Geschichte der Pädagogik. Von der Aufklärung bis zur Gegenwart. Wetzlar: Büchse der Pandora.

Caragliu, A., C. Del Bo \& P. Nijkamp (2011): Smart Cities in Europe. In: Journal of Urban Technology, 18 (2), 65-82.

Dilthey, W. (1982): Das Wesen der Philosophie. (Nachdr. der Ausgabe von 1907). Stuttgart/Göttingen: B.G. Teubner, Vandenhoeck \& Ruprecht.

Dorsch, C., N. Grünberg, O. Wolff \& D. Kanwischer (2016): Mündigkeit und Lehrerbildung in fächer- und phasenübergreifender Perspektive. In: Budke, A. \& M. Kuckuck (Hrsg.): Politische Bildung im Geographieunterricht. (in Druckvorbereitung).

Giddens, A. (1996): Konsequenzen der Moderne. Frankfurt am Main: Suhrkamp.

Greenfield, A. (2013): Against the smart city. A pamphlet. New York: Do projects.

Gruschka, A. (1994): Bürgerliche Kälte und Pädagogik. Moral in Gesellschaft und Erziehung. Wetzlar: Büchse der Pandora.

Gryl, I. \& T. Jekel (2012): Re-centering GI in Secondary Education: Towards a Spatial Citizenship Approach. In: Cartographica, 47 (1), 18-28.

Harley, J.B. (1989): Deconstructing the Map. In: Cartographica, 26 (2), 1-20. 
Heydorn, H.-J. (1980): Ungleichheit für alle. Zur Neufassung des Bildungsbegriffs. Frankfurt am Main: Syndikat Autoren- u. Verl.-Ges.

Hintermann, C. (2010): Schulbücher als Erinnerungsorte der österreichischen Migrationsgeschichte- eine Analyse der Konstruktion von Migrationen und Migrant/innen in GW-Schulbüchern. In: GW-Unterricht 119, 3-18.

Jörissen, B. \& W. Marotzki (2009): Medienbildung - eine Einführung. Theorie - Methoden - Analysen. Bad Heilbrunn: Klinkhardt.

Kaczorowski, W. (2014): Die smarte Stadt - Den digitalen Wandel intelligent gestalten. Handlungsfelder Herausforderungen Strategien. Stuttgart: Richard Boorberg Verlag.

Kant, I. (1803): Über Pädagogik. Herausgegeben und mit einer Vorrede versehen von D. Friedrich Theodor Rink. Königsberg: Friedrich Nicolovius.

Klafki, W. (2007): Neue Studien zur Bildungstheorie und Didaktik. Zeitgemäße Allgemeinbildung und kritischkonstruktive Didaktik. Weinheim: Beltz.

Kößler, H. (1989): Bildung und Identität. In: Kößler, H. \& K. Jacobs (Hrsg.): Identität. Fünf Vorträge. Erlangen: Univ.-Bibliothek Erlangen-Nürnberg, 51-65.

Mandl, B. \& P. Schaner (2012): Der Weg zum Smart Citizen - soziotechnologische Anforderungen an die Stadt der Zukunft. In: Schrenk, M., V. Popovich, P. Zeile \& P. Elisei (Hrsg.): Proceedings REAL CORP 2012, 191199.

Marotzki, W. (2000): Zukunftsdimensionen von Bildung im neuen öffentlichen Raum. In: Marotzki, W., D.M. Meister \& U. Sander (Hrsg.): Zum Bildungswert des Internet. Wiesbaden: VS Verlag für Sozialwissenschaften, 233-258.

Novy, J. (2015): Smart City-Hype: Die Verdummung der Städte? http://www.carta.info/77252/smart-city-hypedie-verdummung-der-staedte (28.01.15).

Pingel, F. (2010): UNESCO guidebook on textbook research and textbook revision. Paris, Braunschweig: UNESCO; Georg Eckert Institute for International Textbook Research.

Roth, H. (1971): Pädagogische Anthropologie. Band 2: Entwicklung und Erziehung. Hannover: Schroedel.

Schieferdecker, I. (2011): Smart Cities. Deutsche Hochtechnologie für die Stadt der Zukunft. Aufgaben und Chancen. München.
Schulze, U., I. Gryl \& D. Kanwischer (2015): Spatial Citizenship - Zur Entwicklung eines Kompetenzstrukturmodells für eine fächerübergreifende Lehrerfortbildung. In: Zeitschrift für Geographiedidaktik 43 (2), 139-164.

Sennett, R. (2012): No one likes a city that's too smart. http://www.theguardian.com/commentisfree/2012/ dec/04/smart-city-rio-songdo-masdar (28.01.15).

Sommer, M. (2007): Mündigkeit. In: Ritter, J. (Hrsg.): Historisches Wörterbuch der Philosophie. 13 Bände; 1971-2007. Band 6. Basel: Schwabe, 225-235.

Uhlenwinkel, A. (2013): Lernen im Geographieunterricht: Trends und Kontroversen. In: Kanwischer, D. (Hrsg.): Geographiedidaktik. Ein Arbeitsbuch zur Gestaltung des Geographieunterrichts ; mit 4 Tabellen. Stuttgart: Borntraeger, 130-140.

VDE - Verband der Elektrotechnik Elektronik Informationstechnik e.V. (2014): VDE-Studie: Die Smart City kommt in 15 Jahren.

Wolff, C. v. (Hrsg.) (1740): Vernünftige Gedanken von dem gesellschaftlichen Leben der Menschen und insonderheit dem gemeinen Wesen. "Deutsche Politik". Bearbeitet, eingeleitet und herausgegeben von Hasso Hofmann. München: Beck.

Untersuchte Curricula und Schulbücher

Bierwirth, J. (2012a): Terra - Erdkunde 1. Gymnasium Hessen. Stuttgart: Klett.

Bierwirth, J. (2012b): Terra - Erdkunde 2. Gymnsium Hessen. Stuttgart: Klett.

Brodengeier, E. \& W. Korby (Hrsg.) (2014): Fundamente Geographie, Oberstufe. Stuttgart: Klett.

Hessisches Kultusministerium (2010): Lehrplan Erdkunde - Gymnasialer Bildungsgang: Jahrgangsstufen $5 \mathrm{G}$ bis $8 \mathrm{G}$ und gymnasiale Oberstufe. HKM.

Hessisches Kultusministerium (2011): Bildungsstandards und Inhaltsfelder - Das neue Kerncurriculum für Hessen Sekundarstufe I - Gymnasium: Erdkunde. HKM.

Jennings, S. \& F. Pauly (2013a): Diercke - Geographie 1. Hessen Gymnasium. Braunschweig: Westermann.

Jennings, S. \& F. Pauly (2013b): Diercke - Geographie 2. Hessen Gymnasium. Braunschweig: Westermann.

Latz, W., F. Scholz \& N. Backhaus (Hrsg.) (2012): Diercke - Geographie. Braunschweig: Westermann. 


\section{Anhang}

Anhang 1: Quantitative Untersuchungsergebnisse aus den curricularen Dokumenten und Schulbüchern nach Kontextualisierung. (d. B. = direkter Bezug, $A .=$ Anknüpfungspunkt)

\begin{tabular}{|c|c|c|c|c|c|c|c|c|c|c|c|c|c|c|c|}
\hline & & \multicolumn{2}{|c|}{ smart } & \multicolumn{2}{|c|}{ intelligent } & \multicolumn{2}{|c|}{ digital } & \multicolumn{2}{|c|}{ partizip } & \multicolumn{2}{|c|}{ beteilig } & \multicolumn{2}{|c|}{$\begin{array}{l}\text { Stadtplanung / } \\
\text { Stadtplaner }\end{array}$} & \multicolumn{2}{|c|}{ mündig } \\
\hline & & d. B. & A. & d. B. & A. & d. B. & A. & d. B. & A. & d. B. & A. & d. B. & A. & d.B. & A \\
\hline \multirow{9}{*}{ 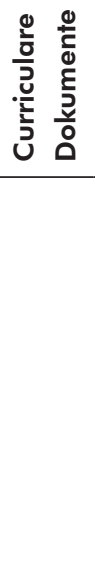 } & Lehrplan & 0 & 0 & 0 & 0 & 0 & 1 & 0 & 0 & 0 & 1 & 0 & 0 & 0 & 0 \\
\hline & Bildungsstandards & 0 & 0 & 0 & 0 & 0 & 0 & 0 & 1 & 0 & 0 & 0 & 0 & 0 & 1 \\
\hline & Klett, & & & & & & & & & & & & & & \\
\hline & Terra 1 & 0 & 0 & 1 & 0 & 0 & 0 & 0 & 0 & 0 & 0 & 0 & 0 & 0 & 0 \\
\hline & (Sek. I) & & & & & & & & & & & & & & \\
\hline & Klett, & & & & & & & & & & & & & & \\
\hline & $\begin{array}{l}\text { Terra } 2 \\
\text { (Sek. I) }\end{array}$ & 0 & 0 & 0 & 0 & 0 & 0 & 0 & 0 & 0 & 0 & 0 & 1 & 0 & 0 \\
\hline & Klett, Fundamente (Sek. II) & 1 & 0 & 3 & 1 & 1 & 0 & 0 & 0 & 0 & 9 & 0 & 4 & 0 & 0 \\
\hline & $\begin{array}{l}\text { Westermann, Diercke } 1 \\
\text { (Sek. I) }\end{array}$ & 0 & 0 & 1 & 0 & 0 & 0 & 0 & 0 & 0 & 0 & 0 & 0 & 0 & 0 \\
\hline \multirow{2}{*}{ 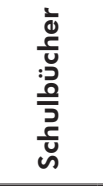 } & $\begin{array}{l}\text { Westermann, Diercke } 2 \\
\text { (Sek. I) }\end{array}$ & 0 & 0 & 1 & 0 & 0 & 0 & 0 & 0 & 0 & 0 & 0 & 0 & 0 & 0 \\
\hline & $\begin{array}{l}\text { Westermann, Diercke } \\
\text { (Sek. II) }\end{array}$ & 1 & 0 & 1 & 0 & 0 & 0 & 0 & 2 & 0 & 5 & 0 & 4 & 0 & 0 \\
\hline \multicolumn{2}{|c|}{ Gesamt } & 2 & 0 & 7 & 1 & 1 & 1 & 0 & 3 & 0 & 15 & 0 & 9 & 0 & 1 \\
\hline
\end{tabular}

